\title{
Nuclease pre-treatment increases efficiency of whole genome sequencing of Influenza B virus in respiratory specimens
}

\author{
Wudtichai Manasatienkij ${ }^{\mathrm{a}, \mathrm{b}}$, Piyawan Chinnawirotpisan ${ }^{\mathrm{b}}$, Weerayuth Kittichotiratc ${ }^{\mathrm{c}}$, Sriluck \\ Simasathien $^{\mathrm{d}}$, Louis R. Macareo ${ }^{\mathrm{b}}$, Damon W. Ellison ${ }^{\mathrm{b}}$, Supapon Cheevadhanarak ${ }^{\mathrm{a}}$, Wiriya \\ Rutvisuttinunt $^{\mathrm{b}}$, Stefan Fernandez ${ }^{\mathrm{b}}$, Chonticha Klungthong ${ }^{\mathrm{b}}$ \\ ${ }^{a}$ Biotechnology Division, School of Bioresources and Technology, King Mongkut's University of Technology Thonburi, Bangkok, Thailand \\ ${ }^{b}$ Department of V irology, Armed Forces Research Institute of Medical Sciences, Bangkok, Thailand \\ 'Systems Biology and Bioinformatics Research Group, Pilot Plant Development and Training Institute, King Mongkut's University of Technology \\ Thonburi, Bangkok, Thailand \\ 'Phramongkutklao Hospital, Bangkok, Thailand
}

Received 2nd July 2019 / Accepted 23rd November 2019

\begin{abstract}
The use of next generation sequencing (NGS) directly on respiratory specimens to obtain viral whole genome sequence (WGS) enhances the capability for rapid and unbiased viral characterization. One of the challenges of using NGS directly in influenza-like illness (ILI) respiratory specimens is the higher proportion of host and bacterial genome compared to viral genetic materials found, which reduces the likelihood of obtaining complete viral genome sequences. This study aims to evaluate nuclease pretreatments prior to sequencing of influenza B virus directly from ILI respiratory specimens. Sequence data were mapped to human, bacteria and influenza B viral genome. In the absence of any nuclease pretreatments, the sequence reads identified as Haemophilus influenzae, Haemophilus parainfluenzae, Neisseria meningitidis and Veillonella parvula were the most prominent genetic materials in respiratory specimens. Filtration followed by nuclease treatment reduced bacterial sequence reads by at least 70 folds in all 4 tested samples, supporting the direct application of NGS in ILI respiratory specimens. Although the pretreatment methods significantly reduced human genome sequences, the remaining human genome especially human rRNA still impact the number and proportion of the viral sequence reads.
\end{abstract}

Keywords: filtration, influenza, Next Generation Sequencing, nuclease, qPCR, respiratory specimen

\section{INTRODUCTION}

Analysis of whole genome sequencing (WGS) provides an in-depth way to study influenza virus, including but not limited to detection of reassortments, genetic drift mutations, antigenic characteristics, and drug resistance (Houldcroft et al., 2017; McGinnis et al., 2016). Next generation sequencing (NGS) is a high throughput sequencing technology and its application in rapid sequencing of viruses is increasing (Houldcroft et al., 2017; McGinnis et al., 2016; Ellison et al., 2016; Stapleford et al., 2016; Radford et al., 2012;
Dimitrov et al., 2017). Virus isolates or PCR products, devoid in great part of the confounding presence of exogenous nucleic acid (NA), greatly facilitates sequencing, unlike direct sequencing from clinical samples. A limitation is that they may introduce biases into the WGS. Direct sequencing from clinical specimens increases the fidelity of the sequences obtained and the sequence depth necessary for the detection of viral population variances and co-infecting pathogens. However, reducing the non-target exogenous NA including

*Author for correspondence: Chonticha Klungthong, Department of Virology, USAMD-AFRIMS 315/6 Ratchawithi Road, Ratchatewi, Bangkok 10400, Thailand. Email - chontichak.fsn@afrims.org 
human and bacterial genome in a clinical sample is costly and time-consuming.

A significant challenge to removing exogenous NA from influenza-like illness (ILI) respiratory samples is the high proportion of host, bacteria, and another genetic materials present (Houldcroft et al., 2017). Hence, nuclease pretreatment protocols for virus enrichment are necessary and often used prior to NA extraction steps (Rosseel et al., 2015; Turber et al., 2009). The MiSeq Illumina platform is often used for sequencing the genome of influenza and other viruses, whether isolates or primary clinical specimens (McGinnis et al., 2016; Laassri et al., 2015; Li et al., 2015; Rutvisuttinunt et al., 2013; Rutvisuttinunt et al., 2015; Parker \& Chen, 2017). Pretreatment protocols prior to NGS have been evaluated using contrived specimens, serum, tissues, and inoculated chicken eggs (Rosseel et al., 2015; Lewandowska et al., 2017; Kohl et al., 2015). Nasopharyngeal swab specimens were also previously tested for evaluation of DNase treatment prior to NGS (Greninger et al., 2010). However, nuclease pretreatment of nasal/throat swab specimens have not been demonstrated.

We have selected influenza B virus positive respiratory specimens for this work because this virus type has been related with very high morbidity and mortality rate in children, adolescents, and immunocompromised individuals (Koutsakos et al., 2016; Wong et al., 2014; Pascua et al., 2017). Influenza B virus, an enveloped RNA virus belonging to the family Orthomyxoviruses which co-circulates with influenza A virus, brings about seasonal epidemics and currently includes two genetically and antigenically distinct lineages, the B/Victoria/2/1987-like and B/Yamagata/16/ 1988-like viruses (Koutsakos et al., 2016). The influenza B viral genome consists of eight segments of negative-sense single-stranded RNA and encodes for at least 11 proteins including three polymerase proteins (PB1, PB2 and PA), three surface glycoproteins (HA, NA and NB), the nucleoprotein (NP), the matrix protein (BM1), the ion channel (BM2), the nonstructural protein (NS), and the nuclear export protein (NEP).

This study aims to evaluate nuclease pretreatments prior to sequencing of influenza B virus directly from ILI respiratory specimens. Respiratory specimens positive for influenza B virus by real-time RT-PCR (qRT-PCR) were extracted and treated with either nuclease alone or filtration followed by nuclease treatment prior to sequencing on Illumina MiSeq. Mapped sequence reads and depth of coverage (DOC) of all 8 genome fragments were used to assess the nuclease pretreatment procedures. An effective nuclease pretreatment would greatly improve the efficiency of molecular surveillance and whole genome sequencing of ILI in determining genetic variation and detection of co-infections.

\section{MATERIALS AND METHODS}

\section{Clinical specimens}

The clinical respiratory specimens used in this study were nasal/throat swab specimens in Universal Transport Media (UTM) (Copan Diagnostics Inc., Corona, USA) collected from sentinel ILI surveillance, part of the Armed Forces Research Institute of Medical Sciences (AFRIMS) influenza surveillance program at Phramongkutklao Hospital (PMK), Bangkok, Thailand. This study was approved by the Institutional Review Boards of PMK and the Walter Reed Army Institute of Research. Written informed consents were obtained from subjects or their parents, if minors. All specimens tested positive for influenza B virus by QuickVue (Quidel, San Diego, USA), and qRT-PCR (Levy et al., 2015). The lab results of influenza testing of the 4 specimens used in this study are shown in Table 1. All samples were aliquoted and stored at $-80^{\circ} \mathrm{C}$. Three aliquots of $280 \mu \mathrm{l}$, each, were used in the study. Lineage of the viral isolate was determined by hemagglutination inhibition assay (HI) (Klungthong et al., 2010).

\section{Pre-treatment methods and RNA extraction}

Two nuclease pretreatment methods were tested here; a 1-step nuclease treatment using DNase I and RNase I, and a 2-step treatment that uses filtration prior to the nuclease treatment. The control for the experiment was one aliquot of each specimen that was processed directly for RNA extraction without pretreatment. For the 1step nuclease treatment, one aliquot of $280 \mu \mathrm{l}$ of each specimen was mixed with $0.1 \mathrm{U} / \mu$ l of Turbo DNase (ThermoFisher, Carlsbad, CA, USA), 0.1 
$\mathrm{U} / \mu \mathrm{l}$ RNase I (ThermoFisher, CA, USA), and $1 \mathrm{X}$ DNase buffer (ThermoFisher, CA, USA). The mixture was incubated at $37^{\circ} \mathrm{C}$ for $90 \mathrm{~min}$ before RNA extraction. For the 2-step nuclease pretreatment method, one aliquot of $280 \mu \mathrm{l}$ of each specimen was filtrated through Spin- $X^{\circledR}$ centrifuge tube filter $0.22 \mu \mathrm{m}$ (Coring, NY, USA) by centrifugation at $14,000 \mathrm{rpm}$ for $3 \mathrm{~min}$. The filtrated specimen was then treated with DNase I and RNase I as previously described prior to RNA extraction. RNA extraction was performed by using QIAamp Viral RNA Mini Kit (QIAGEN, Valencia, CA, USA) in accordance with the manufacturer's instruction.

Table 1. Influenza identification of clinical respiratory specimens.

\begin{tabular}{lcccl}
\hline & \multirow{2}{*}{$\begin{array}{c}\text { Rapid test } \\
\text { (QuickVue) }\end{array}$} & \multicolumn{2}{c}{ Real-time PCR (qPCR) } & \multirow{2}{*}{ Isolation result } \\
\cline { 3 - 4 } & FLU B & Result & Ct & \\
\hline FLU-PMKA2994 & FLU B & FLU B & 19.02 & B Massachusetts \\
FLU-PMKA3009 & FLU B & FLU B & 18.53 & B Massachusetts \\
FLU-PMKA3131 & FLU B & FLU B & 17.73 & B Massachusetts \\
FLU-PMKA3209 & & & B Massachusetts \\
\hline
\end{tabular}

\section{Real-time PCR (qRT-PCR) assays}

The amount of extracted human RNase P (RNP) RNA and influenza B viral RNA from each pretreated sample and control was semiquantitatively analyzed by using qPCR. The human RNP gene was used as a target for the detection of human RNA and non-structural protein (NS) gene was used as a target for detection of the viral RNA. The assays were performed in triplicate by using the SuperScript III Platinum One-Step-qRT-PCR System (ThermoFisher, Carlsbad, CA, USA) with specific primers and probes previously described (Levy et al., 2015; Klungthong et al., 2010). The amplification reactions were performed on Applied Biosystems ${ }^{\circledR} 7500$ fast real-time PCR system (Thermo Fisher Scientific, CA, USA). Each qRT-PCR reaction contained $5 \mu$ of RNA suspension, 20 pmol of each forward and reverse primers, $10 \mathrm{pmol}$ of each probe, $0.5 \mu \mathrm{l}$ of $1: 10$ ROX reference dye, $12.5 \mu \mathrm{l}$ of $2 x \mathrm{xT}-\mathrm{PCR}$ reaction mix, $0.5 \mu \mathrm{l}$ of SuperScript III (ThermoFisher, CA, USA) and $5 \mathrm{U}$ of RNaseOUT (ThermoFisher, CA, USA) in a total volume of $25 \mu \mathrm{l}$. The single-step qRT-PCR consisted of a $30 \mathrm{~min}$ RT step at $50^{\circ} \mathrm{C}, 2 \mathrm{~min}$ of Taq inhibitor inactivation at $95^{\circ} \mathrm{C}$, followed by 45 cycles of PCR at $95^{\circ} \mathrm{C}$ for $15 \mathrm{sec}$ and $55^{\circ} \mathrm{C}$ for 30 sec. The average $\mathrm{Ct}$-values was calculated from Cts obtained from 4 specimens (triplicate reactions/specimen).

\section{DNA library preparation and NGS}

Each extracted RNA sample was concentrated by ethanol precipitation and the quantity of the purified RNA was fluorometrically measured by Qubit $^{\mathrm{TM}}$ RNA HS Assay (ThermoFisher, CA, USA). $0.1-1 \mu \mathrm{g}$ of the RNA from each sample was used for DNA library preparation using TruSeq Stranded Total RNA Library Prep Kit with Ribo-Zero Globin (Illumina, USA), and SuperScript III by following the TruSeq manufacturer's protocol. The concentration and average DNA fragment size of each library were measured by KAPA SYBR ${ }^{\circledR}$ FAST qPCR Kit (KAPA Biosystems, Boston, MA, USA) and High Sensitivity D1000 ScreenTape Assay with Agilent 2200 TapeStation (Agilent Technologies, USA), respectively. The library was pooled and sequenced with $(2 \times 150 \mathrm{bp})$ v2 on MiSeq (Illumina, USA).

\section{NGS data analysis}

Sequencing Analysis Viewer Software (SAV) (Illumina, USA) was used for examining the quality of NGS run. Seventy-eight percent to $91 \%$ of sequence reads analyzed by the SAV had a quality control (QC) base-calling score of $\geq$ Q30. The 150 bp pair-end sequence reads of all samples were demultiplexed by MiSeq Reporter software. FastQC version 0.11 .3 was used to assess the raw sequence reads quality. Nucleotides with quality less than $25(\mathrm{Q}<25)$ at the two ends of each sequence read were trimmed using Trimmomatic (Bolger et al., 2014). Trimmed sequence reads of each sample were analyzed by using Kraken 
(Wood \& Salzberg, 2014) and CLARK (31-mers) (Ounit et al., 2015) in order to identify and estimate the proportion of mapped reads of known bacterial, viral, and human genome sequences. Trimmed sequence reads of each sample were taxonomically classified by using high accuracy Kraken tool (Majid et al., 2015; Wood \& Salzberg, 2014). However, since Kraken does not provide a human database, CLARK (Ounit et al., 2015) was used to identify and estimate the proportion of mapped reads of known bacterial, viral, and human genome sequences. Both CLARK and Kraken have similar strength in precision and sensitivity for bacterial identification with the default settings. The metagenomics were analyzed and graphically visualized by using Krona (Ondov et al., 2011). Figure 1 summarizes the workflow of sequence data analysis.

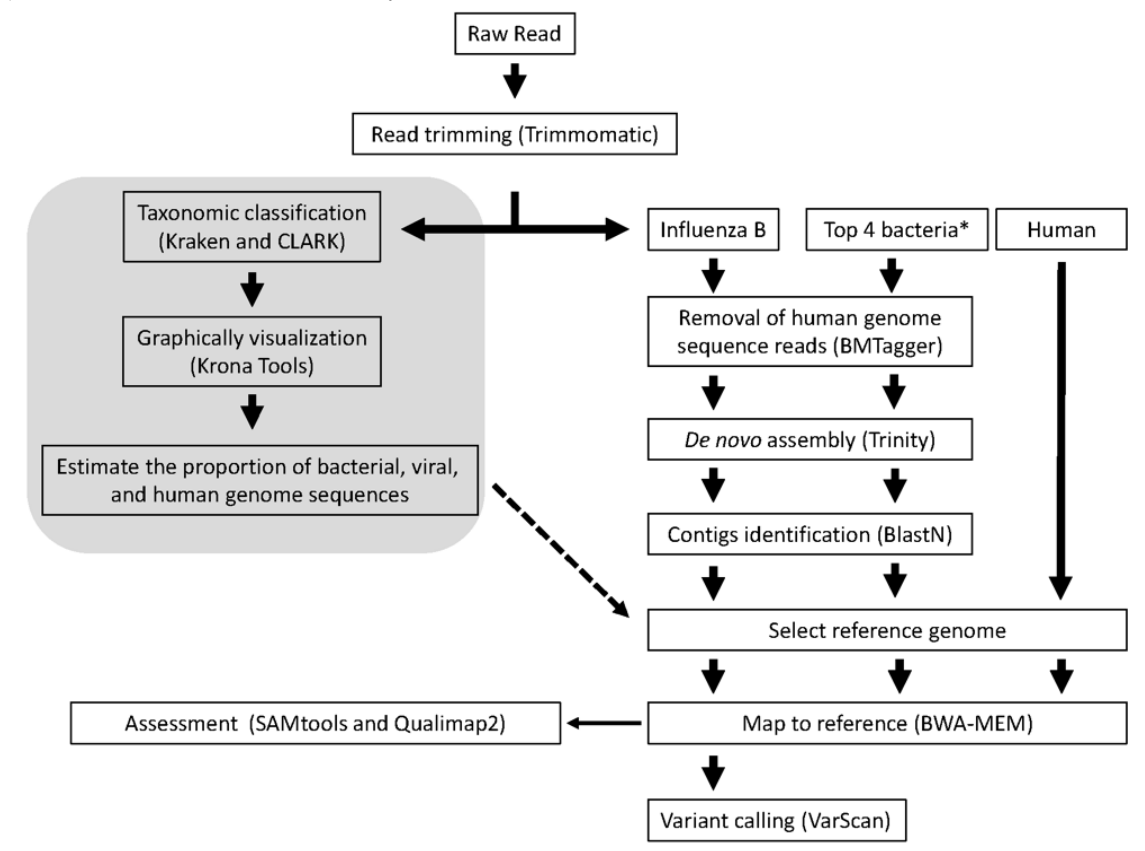

Figure 1. Workflow of sequence data analysis.

*Top 4 bacteria included Haemophilus influenzae, Haemophilus parainfluenzae, Neisseria meningitidis and Veillonella parvula.

\section{Influenza $B$ viral genome sequence analysis}

Removal of the human genome sequences from each sample was performed with the aid of NCBI Best Match Tagger (BMTagger) Human Contamination Screening Tool (ftp://ftp.ncbi.n $\mathrm{lm}$.nih.gov/pub/agarwala/bmtagger/). De novo assembly was performed with the remaining sequence reads after removing human genome sequence using Trinity v.2.2.0 (Grabherr et al., 2011). To select influenza $B$ viral genome reference, the contigs with length of $>200$ nucleotides obtained from de novo assembly were compared with sequences in the GenBank database using BlastN. An $\mathrm{E}$ value of 0.1 was used as the cut-off threshold value for significant hits. The viral complete genome sequence (PB2 $=2,313 \mathrm{bp}, \mathrm{PB} 1=2,259 \mathrm{bp}, \mathrm{PA}=2,181 \mathrm{bp}, \mathrm{HA}$ $=1,755 \mathrm{bp}, \mathrm{NP}=1,683 \mathrm{bp}, \mathrm{NA}=1,408 \mathrm{bp}, \mathrm{M}=$ $1,076 \mathrm{bp}, \mathrm{NS}=1,024 \mathrm{bp}$ ) with the best hit and high identity from BlastN output was evaluated by using Quality Assessment Tool (QUAST) version 4.4 (Gurevich et al., 2013). The viral genome with the highest quality from the assembly was selected and used as the reference genome in the alignment mapping using Burrows-Wheeler Aligner (BWAMEM) (Li \& Durbin, 2009; Fonseca et al., 2012). The sequences of samples FLU-PMKA2994, FLU-PMKA3009, and FLU-PMKA3131 were aligned to referent influenza $B$ viral sequences GENBANK accession number JX513092 (PB2), CY171965 (PB1), LC033024 (PA), CY171959 (HA), CY171786 (NP), LC033227 (NA), LC032932 (M), and LC033229 (NS). The sequences of FLU-PMKA3209 was aligned to referent influenza B viral sequences GENBANK accession number; JX513092 (PB2), LC033207 (PB1), LC033024 (PA), CY171959 (HA), CY171786 (NP), LC033227 (NA), LC032932 (M), 
LC032933 (NS). SAMtools and Qualimap 2 were used to transform the file format, to generate coverage statistics, a consensus sequence, and to evaluate the alignment quality (Heng et al., 2009; Okonechnikov et al., 2015). VarScan was utilized for variant calling on samples that contain at least 10-fold DOC (Koboldt et al., 2009). The numbers of influenza $B$ viral sequence reads and variants obtained from each sample were compared. The statistical significance was evaluated using an unpaired Student's t test. A p value lower than 0.05 was considered statistically significant.

\section{Bacterial and human genome sequence analysis}

The top four bacteria species including Haemophilus influenzae, Haemophilus parainfluenzae, Neisseria meningitidis and Veillonella parvula were identified in all samples using Kraken and CLARK by which the sequence mapped reads of these bacteria genome were found with the highest number and accounted for $\sim 35-54 \%$ of all bacterial sequence reads. The genomes of these bacteria were used as references in the alignment mapping using BWA-MEM and analyzed as representatives of all bacterial genomes in all samples. The numbers of bacterial or human mapped reads obtained from each sample was compared. Human ribosomal RNA (rRNA) was identified using alignment mapping with BWAMEM. Reference human rRNA sequences used in the alignment were obtained from GENBANK including accession numbers: NR146118 (28S rRNA), NR146119 (18S rRNA), NR146120 (5.8S rRNA), NR137295 (mitochondrially 16S rRNA), and NR137294 (mitochondrially 12S rRNA). The number of human rRNA mapped reads obtained from each sample were compared. The statistical significance were evaluated using an unpaired Student's $\mathrm{t}$ test and calculated using Microsoft Excel (2010). A p value that lower than 0.05 was considered statistically significant.

\section{RESULTS}

\section{Human and influenza $B$ viral genome semi- quantification by $q R T-P C R$}

The Ct values obtained from qRT-PCR of RNase $\mathrm{P}$ and NS genes were used to evaluate the amount of human and influenza B viral genome in all samples. The $\mathrm{Ct}$ values are inversely related to the amount of the target within the sample. The average $\mathrm{Ct}$ values of the viral NS genes and human RNase $\mathrm{P}$ genes detection by qRT-PCR in control samples and pretreated samples were shown in Figure 2.

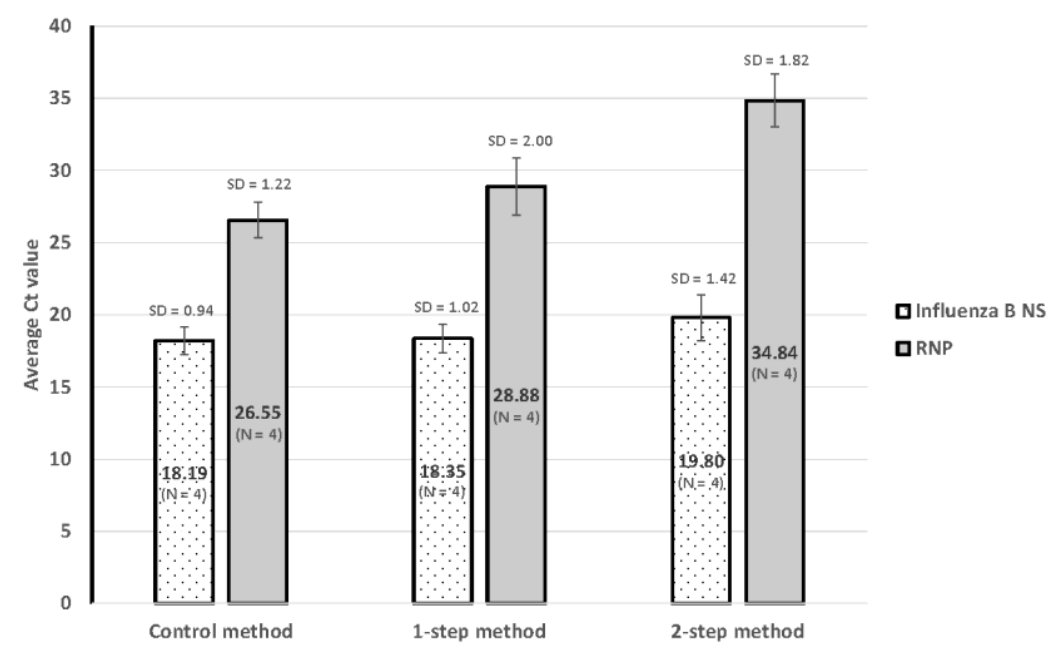

Figure 2. The average $\mathrm{Ct}$ values from influenza B NS and human RNP genes detection by qRT-PCR in the untreated and pretreated samples by 1 -step (nuclease) and 2-step (filter and nuclease) methods. Each average $\mathrm{Ct}$ value obtained from 4 specimens (triplicate reactions/specimen).

Each value was obtained from the average of four specimens (triplicate reactions/specimen). The average Ct value for human RNase P detection in pretreated samples by 1 -step and 2-step methods were significantly higher than that from control samples $(\phi$ value $=0.02$ and 0.003 , respectively) 
and the highest average $\mathrm{Ct}$ was found in pretreated samples by the 2-step method. The average $\mathrm{Ct}$ of the viral NS gene detection in pretreated samples by both methods were not significantly different from control samples $(p$ value $=0.5$ and 0.07 , respectively) and the highest average $\mathrm{Ct}$ was found in pretreated samples by the 2-step method (1.6 Cts different from control). These results indicated that the amount of human RNP gene was reduced significantly when the specimens were treated by both nuclease pretreatment methods, especially by the 2 -step method. In contrast the amount of influenza B viral genome present in the samples was not reduced when treated by both methods. This indicates that both nuclease pretreatment methods had no effect to reduce the amount of the viral genome.

\section{Genome sequences analysis}

The proportion of human, bacterial and influenza $\mathrm{B}$ virus in the control and pretreated samples by 1 -step and 2-step methods analyzed by CLARK are shown in Figure 3.
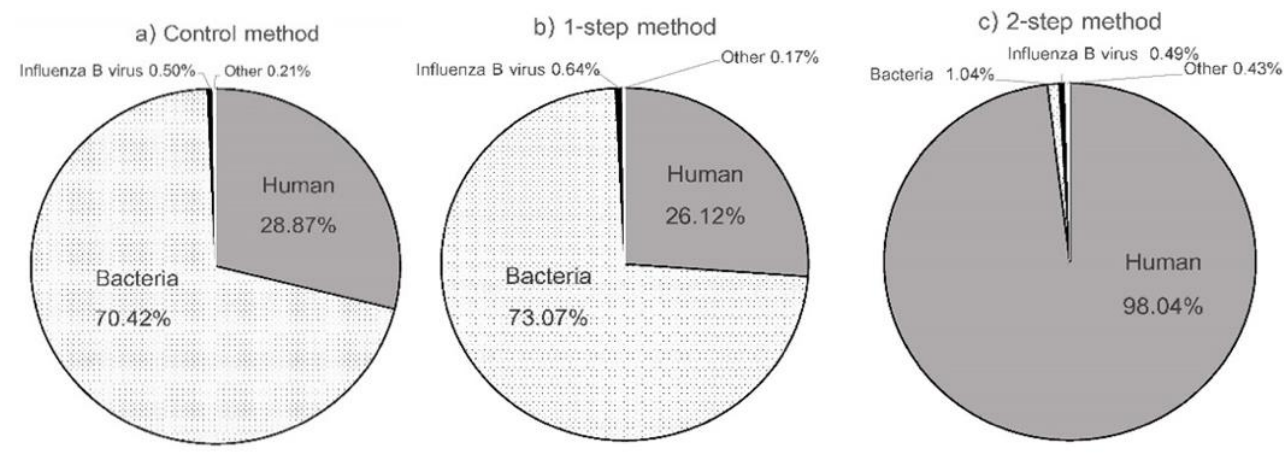

Figure 3. Comparison of the proportion of bacteria, human, influenza B virus and other (unidentified) genome identified in (a) control methods, (b) 1-step method (nuclease) and (c) 2-step method (filter and nuclease). The number of sequence mapped reads of each genome was obtained by using CLARK and BWA-MEM. The percentages shown were the average percentages of sequence mapped reads of bacteria, human, influenza B virus, and other genome obtained from 4 specimens.

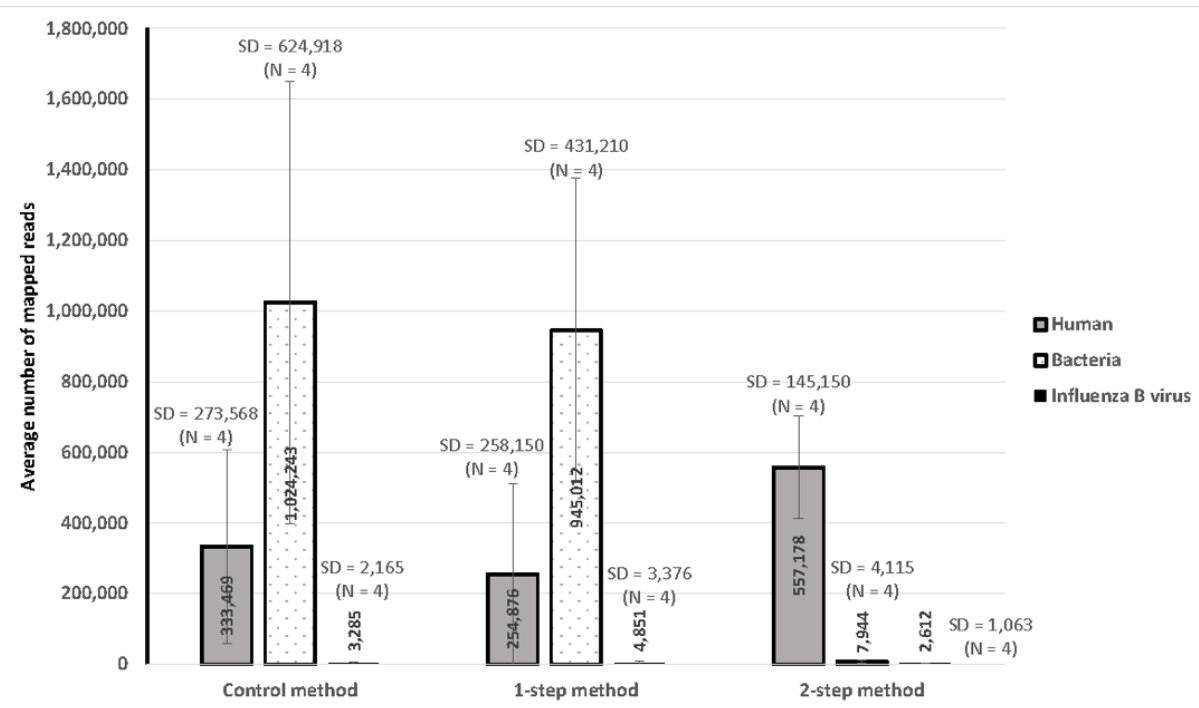

Figure 4. Average number of mapped reads of human, bacteria (Haemophilus influenzae, Haemophilus parainfluenzae, Neisseria meningitidis and Veillonella parvula), and influenza B viral genome in control and samples treated by 1-step (nuclease) and 2 step (filter and nuclease) methods. The number of mapped reads of each genome was obtained by using Kraken. 
Table 2. The total sequence reads, numbers of mapped reads and percentage of mapped sequence reads to human, bacteria, and influenza B viral genome obtained by using Kraken.

\begin{tabular}{|c|c|c|c|c|c|c|c|c|}
\hline \multirow[b]{2}{*}{ Specimen No. } & \multirow[b]{2}{*}{ Pretreatment method } & \multirow{2}{*}{$\begin{array}{c}\text { Total } \\
\text { number } \\
\text { of pair } \\
\text { sequenc } \\
\text { e reads }\end{array}$} & \multicolumn{2}{|c|}{ Human genome } & \multicolumn{2}{|c|}{ Bacteria genome* } & \multicolumn{2}{|c|}{$\begin{array}{c}\text { Influenza B viral } \\
\text { genome }\end{array}$} \\
\hline & & & $\begin{array}{l}\text { Number } \\
\text { of } \\
\text { mapped } \\
\text { reads }\end{array}$ & $\begin{array}{l}\% \text { mapped } \\
\text { reads }\end{array}$ & $\begin{array}{c}\text { Number of } \\
\text { mapped } \\
\text { reads }\end{array}$ & $\begin{array}{c}\% \\
\text { mapped } \\
\text { reads }\end{array}$ & $\begin{array}{l}\text { Number } \\
\text { of } \\
\text { mapped } \\
\text { reads }\end{array}$ & $\begin{array}{c}\% \\
\text { mapped } \\
\text { reads }\end{array}$ \\
\hline \multirow{3}{*}{ FLU-PMKA2994 } & Control (untreated) & $2,594,454$ & 721,912 & 27.83 & $1,671,906$ & 64.44 & 723 & 0.03 \\
\hline & 1-step method (nuclease) & $1,473,136$ & 82,984 & 5.63 & $1,241,796$ & 84.3 & 706 & 0.05 \\
\hline & 2-step (filter and nuclease) & 656,647 & 526,753 & 80.22 & 11,339 & 1.73 & 1,344 & 0.2 \\
\hline \multirow{3}{*}{ FLU-PMKA3009 } & Control (untreated) & 501,237 & 58,351 & 11.64 & 397,335 & 79.27 & 1,900 & 0.38 \\
\hline & 1-step method (nuclease) & 583,953 & 95,131 & 16.29 & 444,389 & 76.1 & 2,397 & 0.41 \\
\hline & 2-step (filter and nuclease) & 758,646 & 693,987 & 91.48 & 12,332 & 1.63 & 2,253 & 0.3 \\
\hline \multirow{3}{*}{ FLU-PMKA3131 } & Control (untreated) & $2,228,272$ & 458,958 & 20.6 & $1,625,990$ & 72.97 & 6,372 & 0.29 \\
\hline & 1-step method (nuclease) & $2,277,735$ & 700,434 & 30.75 & $1,485,275$ & 65.21 & 8,712 & 0.38 \\
\hline & 2-step (filter and nuclease) & 361,989 & 332,270 & 91.79 & 5,876 & 1.62 & 2,569 & 0.71 \\
\hline \multirow{3}{*}{ FLU-PMKA3209 } & Control (untreated) & 569,540 & 94,654 & 16.62 & 401,740 & 70.54 & 4,143 & 0.73 \\
\hline & 1-step method (nuclease) & 821,514 & 140,953 & 17.16 & 608,588 & 74.08 & 7,587 & 0.92 \\
\hline & 2-step (filter and nuclease) & 698,500 & 675,702 & 96.74 & 2,229 & 0.32 & 4,280 & 0.61 \\
\hline
\end{tabular}

* Bacteria genome including top four bacteria species (Haemophilus influenzae, Haemophilus parainfluenzae, Neisseria meningitidis and Veillonella parvula) 
The number of sequence reads, number and percentage of mapped reads including human, bacteria, and influenza B virus of all clinical specimen were obtained by using Kraken and shown in Table 2 from which the average number of mapped reads of human, bacteria, and the viral genome was calculated and plotted in Figure 4. The results showed that the number of mapped reads as well as the percentage of mapped reads of human genome from the samples pretreated using the 2-step method was significantly higher than that from control ( $p$ value $=0.03$ ), while the number from samples pretreated using the 1-step method was not different from control $(p$ value $=$ 0.92). The number and percentage of mapped reads for bacteria from samples pretreated with the 2-step method was significantly lower than from control $(\phi$ value $=2.65 \mathrm{E}-07)$. The number and percentage of mapped reads from samples pretreated with the 1-step method was not statistically different from control $(\phi$ value $=0.60)$. The average number of human and bacteria mapped reads decreased by both nuclease pretreatment methods $(1.13 \mathrm{x}$ by 1 -step method, $2.40 \mathrm{x}$ by 2 -step method). The number and percentage of mapped reads of influenza $B$ viral genome from the samples treated by the 1-step and 2-step methods not different from control $(p$ value $=0.08$ and 0.31 , respectively). However, the ratio of the average number of the viral mapped reads per average number of total mapped reads obtained from samples pretreated by 1-step $(4,851 / 1,204,739=0.004)$ and 2 -step methods $(2,612 / 567,734=0.15)$ were higher than that from control $(3,285 / 1,360,997=0.002)$, respectively. Analysis of human rRNA in all specimens revealed that human rRNA still remained in untreated specimens (16-47\%), pretreated specimens with 1-step method (29$94 \%$ ) and pretreated specimens with 2-step method (66-93\%). We found that most of the human sequence reads $(>50 \%)$ in the 1-step pretreated specimens $(2 / 2)$ and 2-step pretreated specimens (4/4) were human rRNA. Table 3 shows the number and percentage of mapped reads to human $r$ RNA of all samples.

Table 3. The numbers and percentage of mapped reads to human ribosomal RNA.

\begin{tabular}{|c|c|c|c|c|}
\hline \multirow{2}{*}{ Specimen No. } & \multirow{2}{*}{ Pretreatment method } & \multirow{2}{*}{$\begin{array}{l}\text { Total number of } \\
\text { human genome } \\
\text { sequence reads } \\
\text { (pair) }\end{array}$} & \multicolumn{2}{|c|}{ Human rRNA } \\
\hline & & & $\begin{array}{c}\text { Number of } \\
\text { mapped reads } \\
\text { (pair) }\end{array}$ & $\begin{array}{c}\% \\
\text { mapped } \\
\text { reads }\end{array}$ \\
\hline \multirow[t]{3}{*}{ FLU-PMKA2994 } & Control (untreated) & 721,912 & 312,182 & 43 \\
\hline & 1-step method (nuclease) & 82,984 & 24,359 & 29 \\
\hline & 2-step (filter and nuclease) & 526,753 & 393,223 & 75 \\
\hline \multirow[t]{3}{*}{ FLU-PMKA3009 } & Control (untreated) & 58,351 & 9,387 & 16 \\
\hline & 1-step method (nuclease) & 95,131 & 31,403 & 33 \\
\hline & 2-step (filter and nuclease) & 693,987 & 461,344 & 66 \\
\hline \multirow[t]{3}{*}{ FLU-PMKA3131 } & Control (untreated) & 458,958 & 125,588 & 27 \\
\hline & 1-step method (nuclease) & 700,434 & 509,325 & 73 \\
\hline & 2-step (filter and nuclease) & 332,270 & 224,133 & 67 \\
\hline \multirow[t]{3}{*}{ FLU-PMKA3209 } & Control (untreated) & 94,654 & 44,151 & 47 \\
\hline & 1-step method (nuclease) & 140,953 & 132,380 & 94 \\
\hline & 2-step (filter and nuclease) & 675,702 & 629,084 & 93 \\
\hline
\end{tabular}

The quality of influenza B viral genome sequence obtained from control and pretreated samples by both methods was shown in Table 4. We found that the average mean depth of coverage (DOC) for the two control samples with lower viral loads
(Cts 18.57 and 19.57) were 6 times (181.5/31) lower than that of the two control samples with higher viral loads (Cts 17.31 and 17.33). Average mean DOC of all samples pretreated with the 1step method was 1.48 times $(158 / 106.25)$ higher 
than that of control samples. The mean DOC for all samples pretreated with 2-step method was 1.23 times (106.25/86.5) less than that of control samples. The number of segments with complete coding sequence (CDS) of influenza $B$ viral genome increased in samples with $\mathrm{Cts}>18$ treated with both methods. For the untreated samples with $\mathrm{Ct}>18$, the sequences of PB2, HA, and NA of FLU-PMKA2994 and NA of FLUPMKA3009 were found with incomplete CDS. The number of complete CDS segments did not change in the samples with Cts $<18$ treated with either method. Due to DOC falling below the cut-off limit in some regions of the sequences, the results from VarScan were inconclusive.

Table 4. Quality of influenza B viral genome sequence obtained from control and pretreated samples by both methods.

\begin{tabular}{|c|c|c|c|c|c|}
\hline Specimen No. & $\begin{array}{c}\text { Average Cts } \\
\text { of control } \\
\text { (untreated } \\
\text { sample) }\end{array}$ & Pretreatment method & $\begin{array}{l}\text { Mean } \\
\text { DOC } \\
\text { (reads) }\end{array}$ & $\begin{array}{l}\text { Complete } \\
\text { CDS } \\
\text { (segment) }\end{array}$ & $\begin{array}{l}\text { Segment with } \\
\text { incomplete } \\
\text { CDS }\end{array}$ \\
\hline \multirow{3}{*}{$\begin{array}{c}\text { FLU- } \\
\text { PMKA2994 }\end{array}$} & \multirow{3}{*}{18.57} & Control (untreated) & 23 & 5 & PB2, HA, NA \\
\hline & & 1-step (nuclease) & 24 & 8 & none \\
\hline & & 2-step (filter and nuclease) & 47 & 8 & none \\
\hline \multirow{3}{*}{$\begin{array}{c}\text { FLU- } \\
\text { PMKA3009 }\end{array}$} & \multirow{3}{*}{19.57} & Control (untreated) & 39 & 7 & NA \\
\hline & & 1-step (nuclease) & 75 & 8 & none \\
\hline & & 2-step (filter and nuclease) & 75 & 8 & none \\
\hline \multirow{3}{*}{$\begin{array}{c}\text { FLU- } \\
\text { PMKA3131 }\end{array}$} & \multirow{3}{*}{17.33} & Control (untreated) & 210 & 8 & none \\
\hline & & 1-step (nuclease) & 282 & 8 & none \\
\hline & & 2-step (filter and nuclease) & 82 & 8 & none \\
\hline \multirow{3}{*}{$\begin{array}{c}\text { FLU- } \\
\text { PMKA3209 }\end{array}$} & \multirow{3}{*}{17.31} & Control (untreated) & 153 & 8 & none \\
\hline & & 1-step (nuclease) & 251 & 8 & none \\
\hline & & 2-step (filter and nuclease) & 142 & 8 & none \\
\hline
\end{tabular}

DOC: depth of coverage; CDS: coding sequence.

\section{DISCUSSION}

This study compared two simple nuclease pretreatment methods including a 1 -step method, nuclease treatment, and a 2 -step method, filtration followed by nuclease treatment, applied prior to RNAseq NGS library preparation for WGS. Nasal/throat swab specimens collected from subjects with acute ILI were selected to test these treatment methods because they are routinely found with both human and bacterial genomes (Rosseel et al., 2015). Our results confirmed that the highest contributor of background NA content in the untreated nasal/throat swab specimens was bacteria followed by human. In contrast, other reports indicate that human genome contribution to background NA in untreated nasopharyngeal swab specimens can reach $>75 \%$ of the obtained sequences (Greninger et al., 2010). Reducing bacteria and human NA in specimens should improve detection and sequencing of the viral genome. We found that the most effective method to reduce bacteria was filtration followed by nuclease treatment (2-step method). Although filtration treatment alone was not examined in this study due to insufficient volume of the specimens, we expected that the filtration step in the 2-step method was effective in reducing bacterial sequence reads. From our results, nuclease treatment alone did not reduce bacterial genome. This is consistent to a prior report showing that DNase treated nasopharyngeal swab specimens prior to NGS still provided high amount of bacterial rRNA (Greninger et al., 2010).

Nuclease treatment has previously been reported as effective for host NA removal (Hall et 
al., 2014; Dimitrov et al., 2017). Here, we quantitated the reduction in contaminant human genome by nuclease treatment (1-step treatment) of 4 specimens (measured by the increasing Cts in the amplification of the human RNP gene and the decrease in the number of human sequence reads shown in sequencing). The significant reduction of human sequence reads were observed mainly in specimen FLU-PMK2994 and not the others. In addition, we observed that using the 2-step treatment substantially increased the number of human sequence reads. The relative increase in human sequence reads might have resulted from the reduction of bacteria genome in the filtering process. This implies that reducing bacterial sequence reads by filtration alone may not be able to improve the sequencing of influenza $\mathrm{B}$ viral genome as well since the high amount of human genome still remained. Technically, filtration is not able to reduce particles smaller than $0.22 \mu \mathrm{m}$, for instance, exogenous NA which may have increased during the freeze/thaw processing of samples prior to initial extraction (Hall et al., 2014).

We observed slight decrease in the amount of influenza B viral genome when using either nuclease pretreatment method, as measured by qRT-PCR. In the specimens tested, where the virus was found at Cts of 17 to19, removal of free extracellular viral genome by nuclease treatment and intracellular virus genome by filtration did not affect the amount of the viral genome detected. The sequencing results also showed that both nuclease pretreatment methods did not reduce the number of influenza B mapped reads. However, the nuclease pretreatment methods increased the ratio of the average number of influenza $B$ mapped reads per average number of total mapped reads. This likely is due to decreasing background of non-viral NA and increasing the available viral NA.

The WGS via RNAseq typically has lower sensitivity than amplicon-sequencing (targeted sequencing with influenza specific primers). With our original RNAseq protocol without nuclease pretreatments, the cut-off for obtaining complete genome is a Ct of 18 (Rutvisuttinunt et al., 2013; Rutvisuttinunt et al., 2015). To test whether enrichment would increase the sequencing sensitivity, we selected samples around the cut-off viremia for the experiment. If the enrichment improves the sensitivity of the protocol, larger genome coverage and possibly higher DOC should be obtained when compared to the no treatment condition. We observed that the specimens with lower viral load (Cts 18.57 and 19.57) showed mean DOC 6-fold less than that of the specimens with higher viral load (Cts 17.31 and 17.33). The 1-step method was able to improve the mean DOC of influenza B viral genome (1.48 fold increase), but the 2-step method reduced the mean DOC 1.23 fold. We found the 2-step method to be inconsistent with regard to increasing and decreasing the mean DOC in individual samples. This might be the result of unequal removal of intracellular viral genetic materials from each specimen by filtration and/or random technical error (such as pipetting), likely to occur more frequently in the 2-step than in the 1-step procedure. Increased number of complete CDS segments were found in only two specimens with lower viral loads. This indicated that the quality of the viral genome sequences obtained from specimens with lower viral loads was improved by either nuclease pretreatment method. Although the background of human and bacteria NA was decreased by both nuclease pretreatment methods, the amount of specific viral genome existing in the clinical specimen is still a critical factor affecting the quality and quantity of viral genome sequences obtained from sequencing. This is consistent with a previous report showing a high correlation of influenza viral load to quantity of influenza sequences obtained from NGS (Fischer et al., 2015). From this study we were able to obtain influenza $B$ viral complete genome sequences directly from respiratory specimens using nuclease pretreatment (specimens with Cts 17.31 to 19.57 ). However, the cut off $\mathrm{Ct}$ value of the influenza $\mathrm{B}$ viral specimen for sequencing is still unclear. The study by Rutvisuttinunt et al., 2015, also showed successful metagenomics sequencing with 2-step nuclease pretreatment (centrifugation followed by DNase I treatment) directly from respiratory specimens with Cts value for influenza B 22.45 and 22.18, although the incomplete genome with DOCs from this study were very low (17.5 and 3.1, respectively). Further testing with more clinical specimens containing limited viral RNA or weak positive specimens $(\mathrm{Ct}>20)$ may provide the cut off $\mathrm{Ct}$ value and more clarification. With 
Illumina MiSeq capacity, reducing the number of specimens per sequencing run is one feasibly option to increase the quality and quantity for viral genome sequencing directly from clinical specimens, especially from those with low viral loads, but this will also increase the cost of sequencing per specimen.

As expected, the consensus sequences of influenza B viral complete genome obtained from individual specimens pretreated with both methods showed 100\% identity with those untreated specimens. This demonstrates that pretreatment methods did not change the nucleotide consensus sequences of viruses in pretreated specimens. Nucleotide heterogeneity (variants) in the viral sequences from each individual specimen (untreated and pretreated by both methods) analyzed by using VarScan yielded inconclusive results due to low DOC in some samples (mean DOC <100). It is still unclear whether or not applying pretreatment methods such as filtration that technically remove intracellular viral genome will introduce bias on viral variants analysis. Comparison between intracellular variants (in the cells that cannot pass through the filter) and extracellular variants (in the filtrate) may clarify this query. Nucleotide heterogeneity of influenza $B$ virus in clinical specimens was previously examined and reported, yielding conflicting findings. Rutvisuttinunt et al., 2015 found that influenza B virus in clinical specimens contained more variants than influenza A/H3N2 and A/H1N1. Varying bioinformatics methods and interpretation criteria may cause different findings on this type of analysis.

\section{CONCLUSION}

Currently, the use of unbiased non-targeted metagenomics RNA sequencing allows WGS of known as well as unknown viral pathogens to be obtained. However, eliminating host genomic materials without impacting pathogen genomic material has been challenging. Here, we evaluated two pretreatment methods prior to NGS directly on respiratory specimens to obtain influenza $\mathrm{B}$ viral whole genome sequences. The two pretreatment methods included 1) nuclease treatment and 2) filtration followed by nuclease treatment. We found that bacterial genetic material was the predominant genetic materials in the nasal/throat swab specimen. We were able to obtain influenza B viral whole genome sequences directly from specimens pretreated with either method. However, selection of pretreatment methods should be based on type of specimen and purposes of sequence analysis.

\section{ACKNOWLEDGEMENTS}

The authors wish to acknowledge the contributions of clinical, laboratory, and administrative personnel at AFRIMS and PMK hospital. We thank the study coordinators at AFRIMS for their support of the project. We are grateful to the patients for their participation in the surveillance program.

This research was funded by a grant from the Armed Forces Health Surveillance Center-Global Emerging Infections Surveillance and Response System. The funders had no role in study design, data collection and analysis, decision to publish, or preparation of the manuscript. Material has been reviewed by the Walter Reed Army Institute of Research. There is no objection to its presentation and/or publication. The opinions or assertions contained herein are the private views of the author, and are not to be construed as official, or as reflecting true views of the Department of the Army or the Department of Defense. The investigators have adhered to the policies for protection of human subjects as prescribed in AR 70-25.

\section{REFERENCES}

Bolger, A. M., Lohse, M., \& Usadel, B. 2014. Trimmomatic: a flexible trimmer for Illumina sequence data. Bioinformatics 30(15): 2114-2120.

Dimitrov, K.M., Sharma, P., Volkening, J.D., Goraichuk, I.V., Wajid, A., Rehmani, S.F., Basharat, A., Shittu, I., Joannis, T.M., Miller, P.J., \& Afonso, C.L. 2017. A robust and costeffective approach to sequence and analyze complete genomes of small RNA viruses. Virology Journal 14(1): 72.

Ellison, D.W., Ladner, J.T., Buathong, R., Alera, M.T., Wiley, M.R., Hermann, L., Rutvisuttinunt, W., Klungthong, C., Chinnawirotpisan, P., Manasatienkij, W., \& Melendrez, M.C. 2016. Complete Genome Sequences of Zika Virus Strains Isolated from the Blood of Patients in Thailand in 
2014 and the Philippines in 2012. Genome Announcements 4(3): e00359-16.

Fischer, N., Indenbirken, D., Meyer, T., Lütgehetmann, M., Lellek, H., Spohn, M., Aepfelbacher, M., Alawi, M., \& Grundhoff, A. 2015. Evaluation of unbiased next-generation sequencing of RNA (RNA-seq) as a diagnostic method in influenza virus-positive respiratory samples. Journal of Clinical Microbiology 53(7): 2238-2250.

Fonseca, N.A., Rung, J., Brazma, A., \& Marioni, J.C. 2012. Tools for mapping high-throughput sequencing data. Bioinformatics 28(24): 3169-3177.

Grabherr, M.G., Haas, B.J., Yassour, M., Levin, J.Z., Thompson, D.A., Amit, I., Adiconis, X., Fan, L., Raychowdhury, R. Zeng, Q., \& Chen, Z. 2011. Trinity: reconstructing a fulllength transcriptome without a genome from RNA-Seq data. Nature Biotechnology 29(7): 644-652.

Greninger, A.L., Chen, E.C., Sittler, T., Scheinerman, A., Roubinian, N., Yu, G., Kim, E., Pillai, D.R., Guyard, C., Mazzulli, T., \& Isa, P. 2010. A metagenomic analysis of pandemic influenza A (2009 H1N1) infection in patients from North America. PloS one 5(10): e13381.

Gurevich, A., Saveliev, V., Vyahhi, N., \& Tesler, G. 2013. QUAST: quality assessment tool for genome assemblies. Bioinformatics 29(8): 1072-1075.

Hall, R.J., Wang, J., Todd, A.K., Bissielo, A.B., Yen, S., Strydom, H., Moore, N.E., Ren, X., Huang, Q.S., Carter, P.E., \& Peacey, M. 2014. Evaluation of rapid and simple techniques for the enrichment of viruses prior to metagenomic virus discovery. Journal of Virological Methods 195: 194-204.

Heng, L., Handsaker, B., Wysoker, A., Fennell, T., \& Ruan, J. 2009. 1000 Genome Project Data Processing Subgroup. The sequence align-ment/map format and samtools. Bioinformatics 25(16): 2078-2079.

Houldcroft, C.J., Beale, M.A., \& Breuer, J. 2017. Clinical and biological insights from viral genome sequencing. Nature Reviews Microbiology 15(3): 183-192.

Klungthong, C., Chinnawirotpisan, P., Hussem, K., Phonpakobsin, T., Manasatienkij, W., Ajariyakhajorn, C., Rungrojcharoenkit, K., Gibbons, R.V., \& Jarman, R.G. 2010. The impact of primer and probe-template mismatches on the sensitivity of pandemic influenza A/H1N1/2009 virus detection by real-time RTPCR. Journal of Clinical Virology 48(2): 91-95.

Koboldt, D.C., Chen, K., Wylie, T., Larson, D.E., McLellan, M.D., Mardis, E.R., Weinstock, G.M., Wilson, R.K., \& Ding, L. 2009. VarScan: variant detection in massively parallel sequencing of individual and pooled samples. Bioinformatics 25(17): 2283-2285.

Kohl, C., Brinkmann, A., Dabrowski, P.W., Radonić, A., Nitsche, A., \& Kurth, A. 2015. Protocol for metagenomic virus detection in clinical specimens. Emerging Infectious Diseases 21(1): 48-57.

Koutsakos, M., Nguyen, T.H., Barclay, W.S., \& Kedzierska, K. 2016. Knowns and unknowns of influenza B viruses. Future Microbiology 11(1): 119-135.

Levy, J.W., Bhoomiboonchoo, P., Simasathien, S., Salje, H., Huang, A., Rangsin, R., Jarman, R.G., Fernandez, S., Klungthong, C., Hussem, K., \& Gibbons, R.V. 2015. Elevated transmission of upper respiratory illness among new recruits in military barracks in Thailand. Influenza and Other Respiratory Viruses 9(6): 308-314.

Lewandowska, D.W., Zagordi, O., Geissberger, F.D., Kufner, V., Schmutz, S., Böni, J., Metzner, K.J., Trkola, A., \& Huber, M. 2017. Optimization and validation of sample preparation for metagenomic sequencing of viruses in clinical samples. Microbiome 5(1): 94-106.
Li, H., \& Durbin, R. 2009. Fast and accurate short read alignment with Burrows-Wheeler transform. Bioinformatics 25(14): 1754-1760.

Li, L., Deng, X., Mee, E.T., Collot-Teixeira, S., Anderson, R., Schepelmann, S., Minor, P.D., \& Delwart, E. 2015. Comparing viral metagenomics methods using a highly multiplexed human viral pathogens reagent. Journal of Virological Methods 213: 139-146.

Lindgreen, S., Adair, K.L., \& Gardner, P.P. 2016. An evaluation of the accuracy and speed of metagenome analysis tools. Scientific Reports 6: 19233.

Majid, L., Zagorodnyaya, T., Plant, E.P., Petrovskaya, S., Bidzhieva, B., Ye, Z., Simonyan, V., \& Chumakov, K. 2015. Deep sequencing for evaluation of genetic stability of influenza A/California/07/2009 (H1N1) vaccine viruses. PloS One 10(9): e0138650.

McGinnis, J., Laplante, J., Shudt, M., \& George, K.S. 2016. Next generation sequencing for whole genome analysis and surveillance of influenza A viruses. Journal of Clinical Virology 79: 44-50.

Okonechnikov, K., Conesa, A., \& García-Alcalde, F. 2015. Qualimap 2: advanced multi-sample quality control for high-throughput sequencing data. Bioinformatics 32(2): 292294.

Ondov, B.D., Bergman, N.H., \& Phillippy, A.M. 2011. Interactive metagenomic visualization in a Web browser. BMC Bioinformatics 12(1): 385.

Ounit, R., Wanamaker, S., Close, T.J., \& Lonardi, S. 2015. CLARK: fast and accurate classification of metagenomic and genomic sequences using discriminative k-mers. $B M C$ Genomics 16(1): 236.

Parker, J., \& Chen, J. 2017. Application of next generation sequencing for the detection of human viral pathogens in clinical specimens. Journal of Clinical Virology 86: 20-26.

Pascua, P.N.Q., Mostafa, H.H., Marathe, B.M., Vogel, P., Russell, C.J., Webby, R.J., \& Govorkova, E.A. 2017. Pathogenicity and peramivir efficacy in immunocompromised murine models of influenza B virus infection. Scientific Reports 7(1): 7345.

Radford, A.D., Chapman, D., Dixon, L., Chantrey, J., Darby, A.C., \& Hall, N. 2012. Application of next-generation sequencing technologies in virology. The Journal of General Virology $93(\mathrm{Pt}$ 9): 1853-1868.

Rosseel, T., Ozhelvaci, O., Freimanis, G., \& Van Borm, S. 2015. Evaluation of convenient pretreatment protocols for RNA virus metagenomics in serum and tissue samples. Journal of Virological Methods 222: 72-80.

Rutvisuttinunt, W., Chinnawirotpisan, P., Simasathien, S., Shrestha, S.K., Yoon, I.K., Klungthong, C., \& Fernandez, S. 2013. Simultaneous and complete genome sequencing of influenza A and B with high coverage by Illumina MiSeq Platform. Journal of Virological Methods 193(2): 394-404.

Rutvisuttinunt, W., Chinnawirotpisan, P., Thaisomboonsuk, B., Rodpradit, P., Ajariyakhajorn, C., Manasatienkij, W., Simasathien, S., Shrestha, S.K., Yoon, I.K., Klungthong, C., \& Fernandez, S. 2015. Viral subpopulation diversity in influenza virus isolates compared to clinical specimens. Journal of Clinical Virology 68: 16-23.

Stapleford, K.A., Moratorio, G., Henningsson, R., Chen, R., Matheus, S., Enfissi, A., Weissglas-Volkov, D., Isakov, O., Blanc, H., Mounce, B.C., \& Dupont-Rouzeyrol, M. 2016. Whole-genome sequencing analysis from the chikungunya virus Caribbean outbreak reveals novel evolutionary genomic elements. PLoS Neglected Tropical Diseases 10(1): e0004402.

Thurber, R.V., Haynes, M., Breitbart, M., Wegley, L., \& Rohwer, F. 2009. Laboratory procedures to generate viral metagenomes. Nature Protocols 4(4): 470-483. 
Wong, C.K., Tsang, G.C., Chan, K.H., Li, O.T., Peiris, M., \& Poon, L.L. 2014. A novel molecular test for influenza B virus detection and lineage differentiation. Journal of Medical Virology 86(12): 2171-2176.

Wood, D.E., \& Salzberg, S.L. 2014. Kraken: ultrafast metagenomic sequence classification using exact alignments. Genome Biology 15(3): R46. 